\title{
Photonic bandgap fibres for low-latency data transmission
}

D.J. Richardson, Y. Chen, N.V. Wheeler, J.R. Hayes, T. Bradley, Z. Liu, S.R. Sandoghchi, G. T. Jasion, T. Bradley, E. Numkam Fokoua, D.R. Gray, R. Slavik, Y. Jung, N.H.L. Wong, F. Poletti and M.N. Petrovich

\author{
Optoelectronics Research Centre, University of Southampton, Southampton, SO17 1BJ, UK
}

\begin{abstract}
We review progress in the design, fabrication and characterisation of hollow core photonic band gap fibres that have led to the production of low-latency data transmission fibres of $>10 \mathrm{~km}$ length with $200 \mathrm{~nm}$ bandwidth and losses at the $5 \mathrm{~dB} / \mathrm{km}$ level.
\end{abstract}

\section{Introduction}

Renewed interest has developed over recent years in the use of hollow-core photonic bandgap fibres (HC-PBGFs) for applications in communications. The primary reasons for this lie in their potential for low loss (ultimately potentially down to values lower than that of conventional solid SMF (albeit at wavelengths around $2000 \mathrm{~nm})$ ), their ultralow nonlinearity (since the signals propagate predominantly in air), and the low-latency guided-wave data transmission they enable (again since the signals propagate in air) [1]. Low latency in HCPBGFs is of great interest for various timesensitive applications, including: intra and inter data centre interconnection, high performance computing and for bespoke networks serving, for example, the financial sector [2]. Whilst the potential of the technology has been demonstrated in various system experiments these have generally been on fibres of $\sim 1 \mathrm{~km}$ length due the challenges of making long structurally uniform lengths of fibre [3-6]. Until much longer samples become available to test in application trials of realistic scale, industrial/commercial interest in the technology will obviously remain limited.

In this paper we review our recent advances in the fabrication of long lengths of HC-PBGF with characteristics close to, or beyond in some parameters, the current state of the art. These advances have been enabled by the development of various new numerical design, fabrication and characterization tools and have now resulted in production of the first HC-PBGF sample with a length $>10 \mathrm{~km}$. The fibre has already been successfully used in low latency transmission experiments [7].

\section{Fabrication and characterization advances}

HC-PBGFs are generally fabricated using a two stage stack and draw method [1]. In order to make long lengths of fibre it is essential to ensure a highly uniform second stage preform with carefully matched microstructured cane and cladding tube dimensions, accurate and reliable pressurisation of the preform and excellent diameter stability throughout the fibre draw. It is also critical to ensure a high degree of cleanliness throughout the fabrication process as any inadvertent inclusion of contaminants can lead to localised defects causing excess fibre loss and potentially fibre breaks. It is also critically important to understand in detail how preform structure evolves and distorts during the fibre draw process due to fluid dynamics so that the preform needed to obtain a given target fibre structure can be designed (the fibre structure has to be carefully engineered to avoid the presence of surface modes and to obtain both a broad operating bandwidth and low loss $[8,9]$ ). To this end we have developed non-destructive tools to: assess the structure and consistency of preforms [10] and the presence and nature of defects within the final fibre (so that these can be eliminated through improved processes) $[10,11]$; numerical models of the fluid-dynamics of the fibre drawing process [12]; methods to allow rapid real time assessment of fibre structure [13]; and tools to accurately predict the optical performance of fibres from structural images [14].

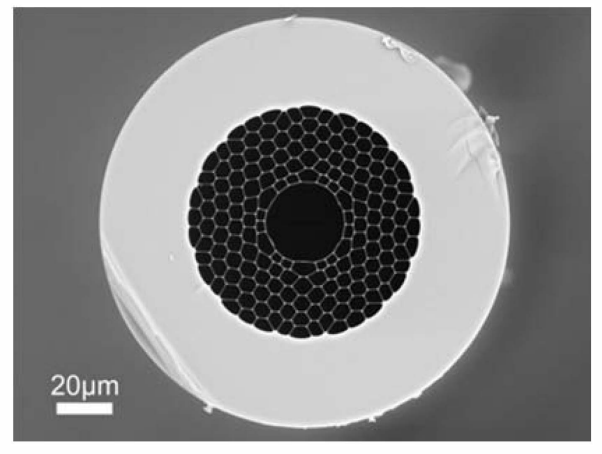

Fig. 1 Cross-sectional SEM image of the 19-cell HC-PBGF.

By applying all of these tools we were able to realise our $11 \mathrm{~km} \mathrm{HC}-\mathrm{PBGF}$ fibre. The HC-PBGF has a 19 cell core and a 5 cladding ring structure (see Fig. 1a). We used a very thin core tube (supplied by OFS Labs, USA) to allow us to increase the length of uniform cane produced in 
the first stage draw and scaled up the yield of the second stage draw to an unprecedented $>11 \mathrm{~km}$ length of high quality fibre. We designed our initial stack to have an optimized ratio between core and cladding strut thickness, thus minimizing any potential surface mode issues associated with the use of a core tube. To further maximize the transmission bandwidth, we targeted extremely high values of air filling factor $(>96 \%)$ of the cladding. The fibre has a $\sim 30 \mu \mathrm{m}$ core diameter, $6.2 \mu \mathrm{m}$ hole-to-hole spacing, a relative hole size $(\mathrm{d} / \Lambda)$ of 0.992 and average cladding strut thickness of just $\sim 50 \mathrm{~nm}$ (measured from high resolution SEM images). Note that previous reports of similarly extreme structures were characterized by high loss $[15$, $16]$, and/or shorter lengths [8].

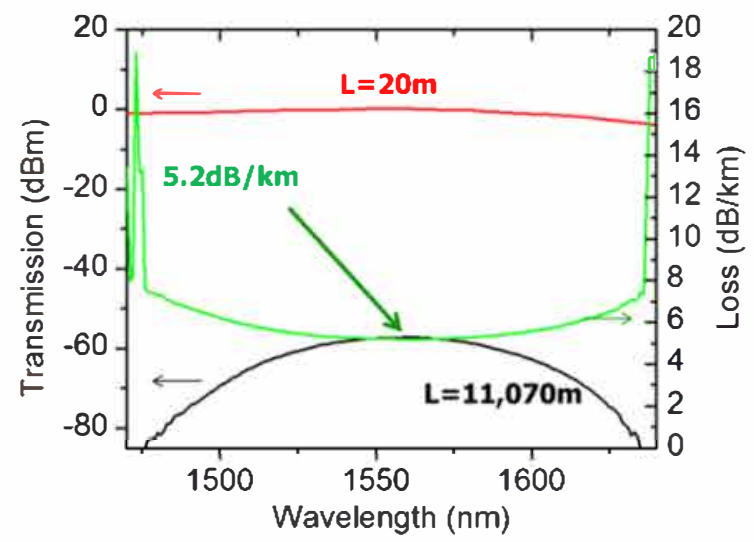

Fig. 2 Spectral transmission (green curve) over the full $11 \mathrm{~km}$ fibre length along with the associated cut-back data.

The transmission loss (Fig. 2), obtained via a cutback measurement $(11.05 \mathrm{~km})$ using a supercontinuum source, shows a minimum value of $5.2 \mathrm{~dB} / \mathrm{km}(1560 \mathrm{~nm})$ with a very flat profile over the measured range $(1475-1635 \mathrm{~nm}$, limited by OSA dynamic range). Simulations from high resolution SEM images predicted a $3 \mathrm{~dB}$ bandwidth in excess of $200 \mathrm{~nm}-$ a value confirmed by cutback measurements from a shorter $800 \mathrm{~m}$ length (which had lesser dynamic range requirements).

To illustrate the uniformity of the fibre we display the relative deviation from target diameter, measured during the fibre draw, which shows $\sim 0.15 \% \quad$ RMS, $\sim 0.7 \%$ peak-to-peak deviation over the full $11 \mathrm{~km}$ length in Fig. $3 a$. We further confirmed the longitudinal consistency of the fibre by coupling light from a $1560 \mathrm{~nm}$ diode laser into the fibre and measuring the total out-scattered power along the length by spooling it at constant speed through an integrating sphere fitted with an InGaAs detector. The scattered power decreased at a constant rate of $\sim 5 \mathrm{~dB} / \mathrm{km}$, as shown in Fig. $3 \mathrm{~b}$, in very good agreement with the lengthaveraged loss value measured via cutback.

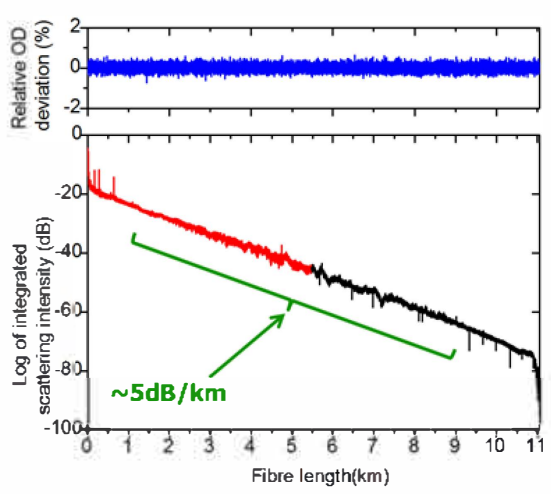

Fig. 3 (a) Relative deviation from target diameter, measured as the fibre was drawn illustrating $0.15 \%$ RMS deviation over the whole $11 \mathrm{~km}$ length. (b) Outscattered power as a function of fiber length indicating a relatively uniform loss of $\sim 5 \mathrm{~dB} / \mathrm{km}$.

\section{Low Latency Transmission Experiment}

We connectorised the PBGF with SMF pigtails (loss $\sim 4 \mathrm{~dB}$ per-pigtail due to the significant mode-mismatch between the fibres (this could be significantly improved with the availability of a suitable buffer fiber with an optimised intermediate core dimension)), obtaining a fully connectorised sample with a total insertion loss of $\approx 66 \mathrm{~dB}$. In order to facilitate transmission we amplified the signal to $\approx 30 \mathrm{dBm}$ prior to launch into the sample - use of such a high power level is acceptable due to the ultra-low nonlinearity of the HC-PBGF. We successfully tested both coherent transmission (20 Gbit/s QPSK) and direct detection (10 Gbit/s) using a low latency compatible OOK signal format. The received signal $(\approx-37 \mathrm{dBm})$ was optically pre-amplified, filtered and finally detected with a 10G $\mathrm{Git} / \mathrm{s}$ receiver.
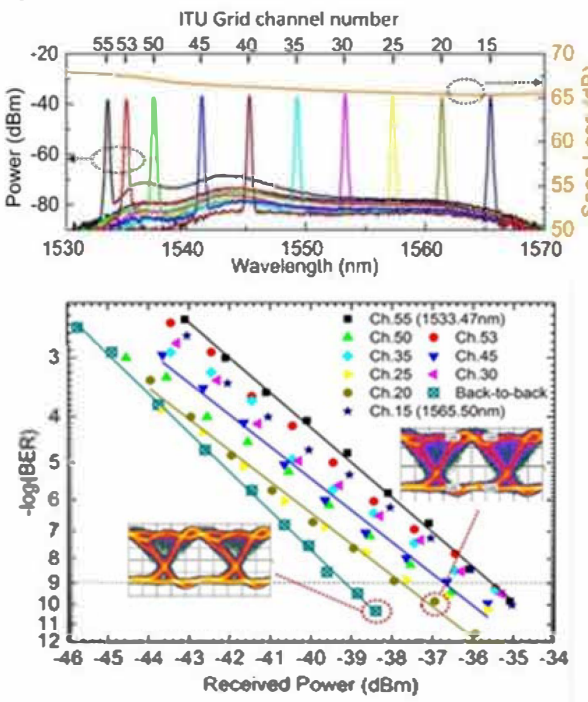

Fig. 4 (a) Channels transmitted through the $11 \mathrm{~km} \mathrm{HC}$-PBGF $(0.1 \mathrm{~nm}$ resolution); (b) OOK BER measurements for the back-to-back and various transmitted channels, and corresponding eye diagrams for the back-to-back and channel 20. 
The optical spectra of various C-band channels (15 to 55) measured at the HC-PBGF output are shown in Fig. 4a. We note that the optical signal-to-noise ratio (OSNR) was $>40 \mathrm{~dB}$ and almost constant over the range $1537-1565 \mathrm{~nm}$. For shorter wavelengths $(1532-1537 \mathrm{~nm})$, the OSNR and thus the overall transmission performance were slightly degraded due to higher levels of ASE from the input EDFA. The corresponding BER performance for the 10 Gbit/s OOK modulation format signal is shown in Fig. 4b. The longer wavelength channels show a BER performance close to that of the $b-2-b$ (penalty $\leq 2 \mathrm{~dB}$ ). A penalty of up to $4 \mathrm{~dB}$ was measured for the short wavelength channels, which we believe to be entirely due to the degraded OSNR. Thus, we conclude that the fibre provides similar performance across the entire C-band with a few $\mathrm{dB}$ penalty that can be attributed to noise generated inside the fibre amplifiers used (two amplifiers with total gain > $70 \mathrm{~dB}$ ) rather than any other effect (e.g. signal degradation due to modal crosstalk).

\section{Conclusions}

In summary we have described the development and transmission performance of a record $11 \mathrm{~km}$ length of HC-PBGF. The fibre has $5 \mathrm{~dB} / \mathrm{km}$ loss and a very broad transmission bandwidth, representing a very significant improvement over the previous state of the art. Data transmission at $10 \mathrm{~Gb} / \mathrm{s}$ along the full $11 \mathrm{~km}$ fibre length without any observable error floor (down to the $10^{-12}$ BER level) has been demonstrated using direct detection, showing only minor penalties and enabling an estimated $>15 \mu \mathrm{s}$ latency reduction relative to the use of a similar physical length of standard fibre. Whilst these results are significant in themselves and bring a number of important applications into range, our (well calibrated) optical and fluid dynamic simulations lead us anticipate significant further reductions in loss (to well below $1 \mathrm{~dB} / \mathrm{km}$ ) $[1,9]$, and the possibility to make significantly longer lengths of fibre per draw [17].

\section{Acknowledgements}

This work was funded by the EU FP7 through project MODE-GAP (grant agreement 258033) and the UK EPSRC through grants EP/H02607X/1 and EP/I061196X (HYPERHIGHWAY).

\section{References}

[1] F. Poletti et al., "Hollow-core photonic bandgap fibers: technology and applications," Nanophotonics, Vol. 2, No. 5-6, p. 315 (2013).
[2] F. Poletti et al., "Towards high-capacity fibre-optic communications at the speed of light in vacuum," Nature Photonics, Vol. 7, No. 4, p. 279 (2013).

[3] V.A.J.M. Sleiffer et al., "30.7 Tb/s $(96 \times 320 \mathrm{~Gb} / \mathrm{s})$ DP32-QAM transmission over 19-cell photonic band gap fiber," Proc. OFC'13, OW11.5, Los Angeles (2013).

[4] V.A.J.M. Sleiffer et al., "High capacity mode-division multiplexed optical transmission in a novel 37-cell hollow-core photonic bandgap fiber," IEEE J. of Lightwave Technology, Vol. 32, No. 4, p. 854 (2014).

[5] R.G.H. van Uden et al., " $8.96 \mathrm{~Tb} / \mathrm{s}(32 \times 28 \mathrm{GBaud} \times$ 32QAM) transmission over $0.95 \mathrm{~km} 19$ cell hollow-core photonic bandgap fiber," Proc. OFC, W4J.3, San Francisco (2014).

[6] B.J. Mangan et al., "First demonstration of hollow-core fiber for intra data center low latency connectivity with a commercial $100 \mathrm{~Gb} / \mathrm{s}$ interface," Proc. OFC, M3D.4, Los Angeles (2015).

[7] Y. Chen et al., "Demonstration of an $11 \mathrm{~km}$ hollow core photonic bandgap fiber for broadband low-latency data transmission," Proc. OFC, Th5A.1, Los Angeles (2015).

[8] R. Amezcua-Correa et al., "Control of surface modes in low loss hollow-core photonic bandgap fibers," Opt. Express 16, 1142-1149 (2008).

[9] E. Numkam Fokoua et al.,"Impact of structura distortions on the performance of hollow-core photonic bandgap fibers," Opt. Express 22, 2735-2744 (2014).

[10] S.R. Sandoghchi et al., "X-ray tomography for structural analysis of microstructured and multimaterial optical fibers and preforms," Opt. Express 22, 26181-26192 (2014).

[11] S.R. Sandoghchi et al., "First investigation of longitudinal defects in hollow core photonic bandgap fibers," Proc. OFC, M2F.6, San Francisco (2014).

[12] G.T. Jasion et al., "MicroStructure Element Method (MSEM): viscous flow model for the virtual draw of microstructured optical fibers," Opt. Express 23, 312329 (2015).

[13] E. Numkam Fokoua et al., "Accurate loss and surface mode modeling in fabricated hollow-core photonic bandgap fibers," Proc. OFC, M2F.5, San Francisco (2014).

[14] E. Numkam Fokoua et al., "Real-time prediction of structural and optical properties of hollow-core photonic bandgap fibers during fabrication," Opt. Letts. 38, 13821384 (2013).

[15] L. Dong et al., "Extending transmission bandwidth of air-core photonic bandgap fibers," Optical Fiber Technol. 16 442-448 (2010).

[16] P.S. Light et al., "Double photonic bandgap hollow-core photonic crystal fiber," Opt. Express 17, 16238-16243 (2009).

[17] G.T. Jasion et al., "Volume manufacturing of hollow core photonic band gap fibers: Challenges and opportunities," Proc. OFC, W2A.37, Los Angeles (2015). 